\title{
Genetic polymorphism in a temporally varying environment: effects of delayed germination or diapause
}

\author{
PHILIP W. HEDRICK \\ Department of Zoology, Arizona State University, Tempe, Arizona 85287, U.S.A.
}

\begin{abstract}
Temporal variation in the environment is generally thought to be less efficient in maintaining genetic polymorphism than spatial variation. However, if there is delayed germination or diapause, in some situations the conditions for genetic polymorphism are greatly broadened in a temporally variable environment. For a model with absolute dominance which means that there can be no type of heterozygous advantage, the upper bound is no longer the geometric mean and the lower bound may not be the arithmetic mean of the fitnesses over environments. In some situations, there is an unstable equilibrium present as well as the stable equilibrium.
\end{abstract}

Keywords: diapause, genetic polymorphism, germination, seed bank, variable environments.

\section{Introduction}

The maintenance of genetic polymorphism in heterogeneous environments continues to be a topic of research interest in evolutionary genetics 40 years after Levene (1953) first gave the conditions for a polymorphism when there is spatial variation in the environment. One conclusion that has been widely accepted over the years is that the conditions for maintenance of polymorphism when there is temporal variation in selection appear to be much more restrictive than those for maintaining variation from spatial variation in fitness (e.g. Hedrick et al., 1976; Hedrick, 1986; Frank \& Slatkin, 1990). Levene (1953) showed that the conditions for polymorphic stability in spatially varying environments are based on the harmonic mean of the fitnesses whereas Dempster (1955) showed that the conditions for temporally varying environments are based on the geometric mean, the latter giving much more stringent limits. Further extensions of the spatial model since then, such as limited gene flow (e.g. Christiansen, 1974) and habitat selection (e.g. Hoekstra et al., 1985; Hedrick, 1990a,b), have shown that these additions may make the conditions for polymorphism with spatial variation even broader.

The difference in the conditions for polymorphism in the two modes of environmental variation can be understood from realizing that all individuals in the traditional temporal model experience all environments, even though in some of these environments particular genotypes may have low fitness. On the other hand, in the spatial model as constructed by Levene, a certain proportion of the population and its descendents exists in the environment for which it has highest fitness and therefore 'escapes' the environments in which it has low fitness. The limited gene flow and habitat selection extensions to the spatial model increase even more the proportion of the population that escapes the environment for which it is not adapted. For example, if there is no gene flow between spatially different environments, then the genotypes adapted to each environment may never experience an environment in which they have low fitness.

Some organisms may be able to avoid an environment for which they are not adapted when there is temporal variation in the environment because they can exist in a life stage that does not encounter the effects of the environment. For example, some plants have extensive seed pools and the seeds that do not germinate do not experience many of the environmental effects encountered by the seeds that do germinate. Likewise, insects or other animals that undergo diapause can avoid environments encountered by individuals that develop without diapause.

Such models have been investigated in an ecological context to determine their influence on the coexistence of competing species. This 'storage' effect (Chesson, 1983) allows each of two competing species to survive environments for which they are not adapted by having a life-history stage that avoids the unfavourable environment. As a result, the conditions for coexistence of the two species are substantially broader allowing coexistence when otherwise it is not possible (e.g. Chesson \& Warner, 1981; Shmida \& Ellner, 1984). In 
related studies, Venable (1989) has examined the significance of temporally variable environments and delayed germination in the evolution of various lifehistory parameters and Seger \& Brockman (1987) have summarized the effects of bet-hedging on fitness in a variety of scenarios.

Recently, Ellner \& Hairston (1994) have extended these ecological models to show that the conditions for maintenance of genetic variation can be greater in fluctuating environments in which there is the opportunity for genotypes to escape unfavourable environments via the storage mechanism. However, it is not clear from their analysis how their findings relate to traditional population genetics models in which specific genotypes are given specific fitnesses and the maintenance of variation at that locus is investigated. Therefore, in the following I will illustrate the potential of the storage effect to influence the conditions for genetic polymorphism in a temporally varying environment when there is such an explicit genetic model.

Investigating the potential effect of seed pools, diapause or related mechanisms on the conditions for polymorphism in a temporally variable environment requires the joint examination of patterns of selection in the environment and patterns of storage in the life stage that allow escape from the effects of given environments. I will examine these phenomena by focusing on a temporal environmental pattern which intuitively would allow the greatest potential for increased polymorphism. More specifically, I will first examine the situation in which there are deterministic cycles in the environment and these cycles coincide with escape in time of unfavourable environments by a proportion of the population. I will then examine a more general model in which the environment varies stochastically. For these models, I will use the absolute dominance model (Prout, 1968), a fitness array in which the relative fitness of only one homozygote varies while the relative fitnesses of the other homozygote and the heterozygote are constant. In this model, there is no possibility for marginal geometric overdominance in which the heterozygote has the highest fitness, such as in the model of Gillespie (1976) (see Hoekstra et al., 1985).

\section{Models and methods}

\section{Cyclic variation in the environment}

Let us assume that there are different environments and that the fitnesses of genotypes $A_{1} A_{1}, A_{1} A_{2}$ and $A_{2} A_{2}$ in environment $k$ are $w_{11 . k}, w_{12 . k}$ and $w_{22 . k}$, respectively. Because I will be examining the absolute dominance model, I will assume that $w_{11 . k}=w_{12 . k}=1$ and that only $w_{22 . k}$, the fitness of genotype $A_{2} A_{2}$, varies. The frequency of the two alleles, $A_{1}$ and $A_{2}$, in generation $t$ are indicated by $p_{t}$ and $q_{t}$, respectively.

Let us assume that a proportion, $g$, of the seeds germinate or animals emerge from diapause in the first generation and that the remaining proportion of the seeds germinate or animals emerge in the next generation. The environment varies in a deterministic cyclic manner alternating between two different environments, 1 and 2, so that the sequence of environments over generations would be $12121212 \ldots$ This sequence of environments and pattern of emergence could occur when there are two generations annually, say spring and fall generations, and most of the individuals do not germinate or emerge in the next generation but remain dormant for one generation. The probability of survival to germinate after one generation of dormancy is $1-d$.

Therefore, the frequency of $A_{1}$ in generation $t$ is

$p_{t}=g^{\prime} p_{t}^{\prime}+\left(1-g^{\prime}\right) p_{t}^{\prime \prime}$,

where

$p_{t}^{\prime}=\left(w_{11 . k} p_{t-1}^{2}+w_{12 . k} p_{t-1} q_{t-1}\right) / w_{t}^{\prime}$

$w_{t}^{\prime}=w_{11 . k} p_{t-1}^{2}+2 w_{12 . k} p_{t-1} q_{t-1}+w_{22 . k} q_{t-1}^{2}$

and

$p_{t}^{\prime \prime}=\left(w_{11 . k} p_{t-2}^{2}+w_{12 . k} p_{t-2} q_{t-2}\right) / w_{t}^{\prime \prime}$

$w_{t}^{\prime \prime}=w_{11 . k} p_{t-2}^{2}+2 w_{12 . k} p_{t-2} q_{t-2}+w_{22 . k} q_{t-2}^{2}$

and

$g^{\prime}=\frac{g w_{t}^{\prime}}{g w_{t}^{\prime}+(1-g)(1-d) w_{t}^{\prime \prime}}$.

The two components of the allele frequency come from seeds or animals generated from the immediately previous generation, $t-1$, and two generations previous, $t-2$. In other words, the allele frequency of individuals 'in storage' varies depending on the selection that their parents encountered. It is assumed that the total number of zygotes is the same each generation and that the proportion of germinating individuals from the two previous generations is weighted by the mean fitness of those progeny.

To determine a protected polymorphism (sensu Prout, 1968), the above equations were iterated for 5000 cycles, 10000 generations, from initial frequencies of 0.0001 and 0.9999 . If the frequency increased from 0.0001 and decreased from 0.9999 , then the situation was a protected polymorphism. By further iteration, the exact equilibrium frequency was determined. 


\section{Stochastic variation in the environment}

To examine the effect of stochastic variation, the same general model as given above was examined except that the pattern of the environment was generated using uniform random numbers. If we assume that there are two environments, then the probability of remaining in environment $k$, given that the present environment is environment $k$, is $\alpha$. The autocorrelation over environments is then $2 \alpha-1$.

Rather than determine whether a particular set of fitness values maintains a polymorphism for a given environmental pattern and germination or emergence schedule, I examined how the environmental pattern influenced the equilibrium distribution of allele frequencies for situations in which the deterministic model above maintained polymorphism. With random variation in the environment, each random pattern of environments will result in a given allele frequency in a given generation. However, if there is a stable polymorphism, then these frequencies, over the different environmental patterns generated by a given autocorrelation and selection scheme, will asymptote at a given frequency distribution. To determine these distributions, preliminary runs of 5000 generations gave the stable equilibrium allele frequency for a given set of fitnesses and given environmental autocorrelation. Then, starting at this equilibrium, 10000 replicates were run for 200 generations to obtain the expected distribution of allele frequencies for a given combination of parameters.

\section{Results}

\section{Cyclic variation in the environment}

Let us begin by examining the region of stability of polymorphism as a function of the mean fitnesses of the variable homozygote in a strictly cyclic environment when there are variable levels of delayed germination or diapause. The region in Fig. 1 between the broken lines is the combination of fitnesses of the $A_{2} A_{2}$ genotype in the two environments that give stability when there is no delayed germination, or diapause, $g=1$. Above the upper broken line, allele $A_{1}$ is lost, $p=0$. Below the lower broken line, which is the arithmetic mean of the two fitness values, $A_{1}$ is fixed, $p=1$. As can be seen, a polymorphism is possible under these conditions but the range is quite narrow when no seeds or animals can avoid the next environment.

When $g=0.75$ or 0.5 , the upper bound is somewhat higher and the region of stability is increased. The lower bound remains the same. For example, if 50 per cent of the seeds or animals avoid the alternative

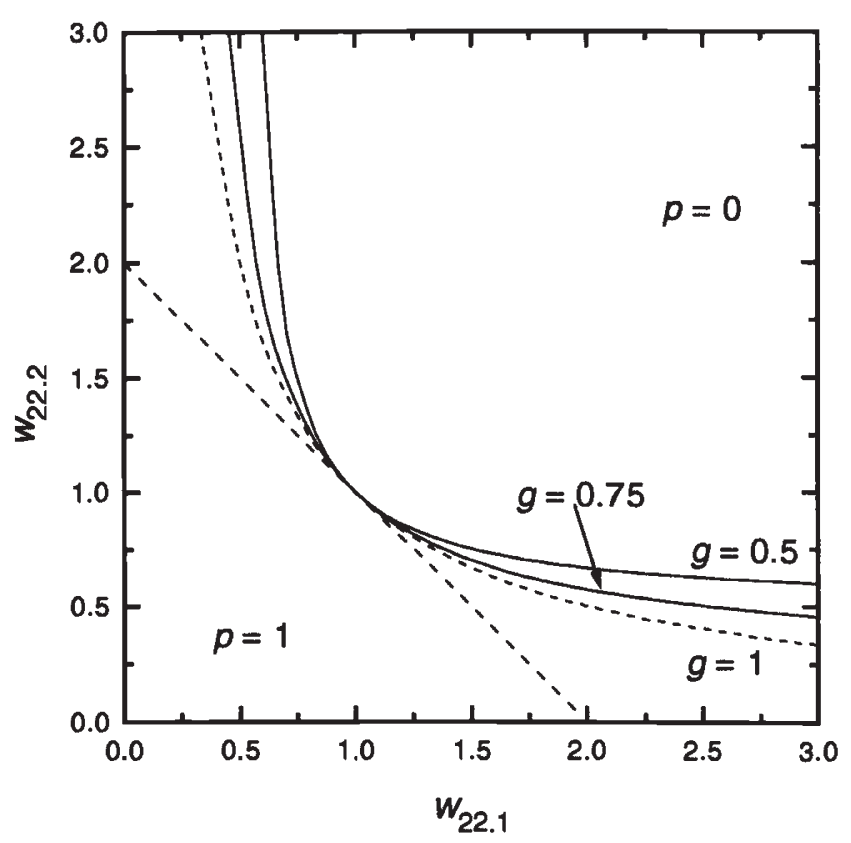

Fig. 1 Region of stability when the fitness of $\mathrm{A}_{2} \mathrm{~A}_{2}$ varies between $w_{22.1}$ and $w_{22.2}$ in a cyclic manner every generation and there are two different levels of delayed germination or diapause. The bounds of the region when there is no delay, $g=1$, are given by the broken lines. The upper bound for levels of $g=0.75$ and $g=0.5$ is given by the solid lines. The regions where allele $A_{1}$ is lost or fixed are indicated by $p=0$ and $p=1$, respectively.

environment, $g=0.5$, and $w_{22.1}=2$, the upper limit is $2 / 3$ while with $g=1$, the upper limit is only $1 / 2$, giving an increase of $1 / 3$ in the range of polymorphism with this storage effect.

When all the seeds are dormant or animals diapause for one generation, $g=0$, then the conditions for a polymorphism are quite broad (solid lines in Fig. 2). In this case, if $w_{22.1}>1$ and $w_{22.2}<1$ or $w_{22.1}<1$ and $w_{22.2}>1$, then there is a polymorphism. Of course, in this limiting case, the populations in the two environments are completely separate and the overall global equilibrium is an average of these two isolated populations. Notice that the arithmetic mean is no longer the lower bound of the region of polymorphism. When $g=0.25$, the conditions for polymorphism are given by the dotted lines in Fig. 2. Notice that here also the lower bound is also reduced so that the total region is greatly increased over that with no delayed germination or diapause as indicated by the broken lines. For example, when $w_{22.1}=1.5$, then there is a stable polymorphism when $w_{22.2}$ is between 0.338 and 0.832 , much larger than the range between 0.5 and $2 / 3$ when $g=1$.

As the level of delayed germination or diapause becomes high ( $g$ becomes low), there may also be an 


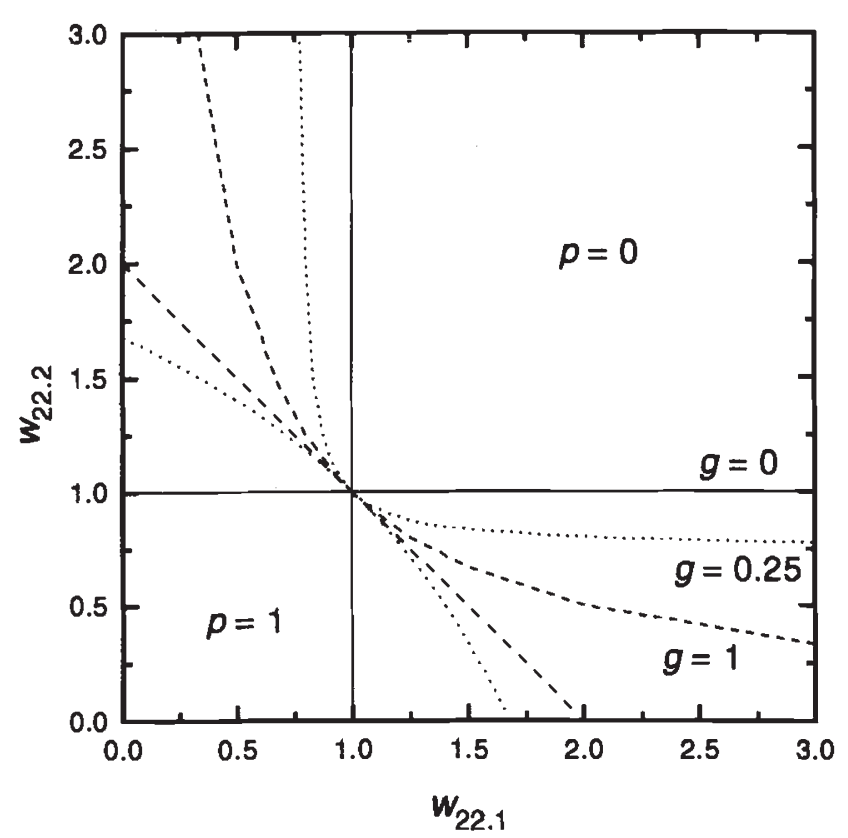

Fig. 2 Region of stability when the fitness of $A_{2} A_{2}$ varies between $w_{22.1}$ and $w_{22.2}$ in a cyclic manner every generation, and the level of delayed germination or diapause is either $g=0.25$ (dotted lines) or $g=0$ (solid lines). Notice that both the upper and lower bounds are different from when there is no delayed germination, $g=1$. The regions where allele $A_{1}$ is lost or fixed are indicated by $p=0$ and $p=1$, respectively.

unstable equilibrium. Figure 3 illustrates this by giving the equilibrium allele frequencies for different levels of $g$ and $w_{22.2}$ when $w_{22.1}=1.5$. As shown for $g=1$ (and $g=0.5$ and 0.75 as well), there is one stable equilibrium that decreases in a nearly linear fashion as $w_{22.2}$ is increased. For $g=0$, there is also a single stable equilibrium of $p=0.5$, which is independent of the value of $w_{22.2}$ (given that it is between 0 and 1). On the other hand, for $g=0.25$ (and other values of $g$ between 0 and slightly above 0.45 ), there are two equilibria, a stable one given by the solid line and an unstable one given by the broken line. For example, if $w_{22.2}=0.4$ and the initial frequency of $A_{1}$ is greater than or equal to 0.895 , the allele frequency will approach 1 . However, this approach to unity is very slow and takes place over thousands of generations. This slow movement is obviously because of the fact that with high $A_{1}$ frequency there are very few $A_{2} A_{2}$ genotypes, the only genotypes that have fitnesses different from unity. On the other hand, if the initial frequency is less than 0.895 , it will approach the stable equilibrium of 0.624 . For this particular set of parameters, these two equilibria converge at $w_{22.2}=0.338$.

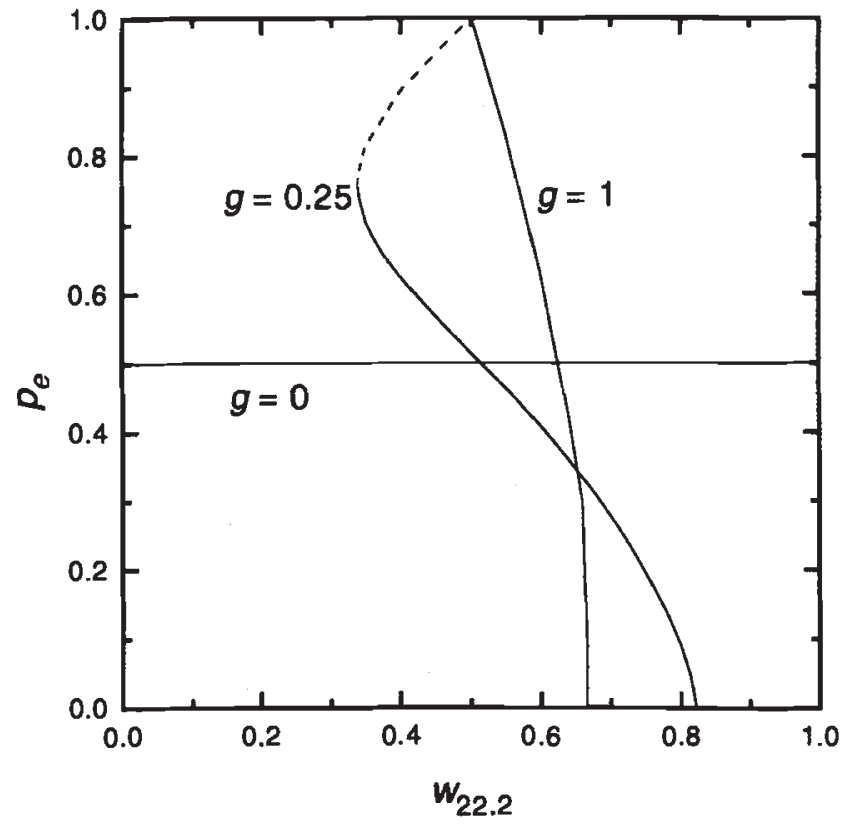

Fig. 3 Equilibrium frequency of $A_{1}$ when $g=0,0.25$ or 1 and $w_{22.1}=1.5$. The stable equilibria are given by solid lines and the unstable equilibria for $g=0.25$ are indicated by the broken line.

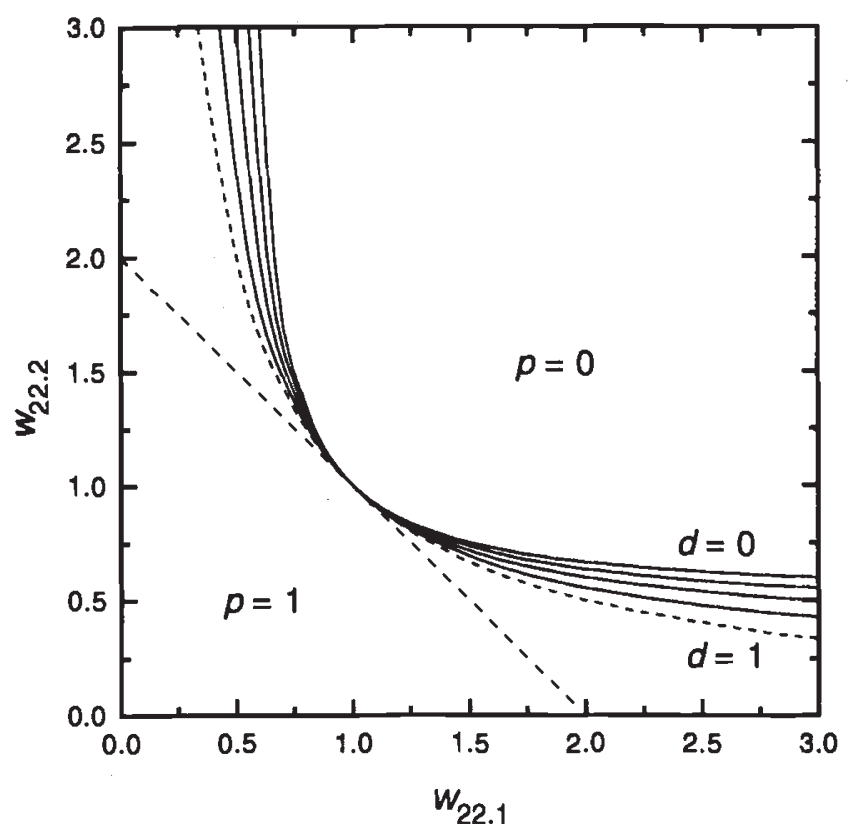

Fig. 4 Region of stability when the fitness of $A_{2} A_{2}$ varies between $w_{22.1}$ and $w_{22.2}$ in a cyclic manner every generation, and the level of delayed germination or diapause is $g=0.5$, and there are different levels of mortality during the dormancy or diapause period. The upper bound of the region is given by the solid lines and the lower bound by the straight, broken line. The unlabelled lines have mortality levels of $0.75,0.5$ and 0.25 , respectively, reading from the bottom to the top. The regions where allele $A_{1}$ is lost or fixed are indicated by $p=0$ and $p=1$, respectively. 
While the seeds are dormant or animals diapause, there may be a significant chance that they do not survive. Figure 4 gives the region for stability when the level of mortality during the dormancy period varies from 0 per cent, $d=0$, to 100 per cent, $d=1$, when the proportion of germination is $g=0.5$. Notice that the limits for $d=1$ and $d=0$ are the same as given in Fig. 1 for $g=1$ and $g=0.5$. Overall, the stability limits are reduced by mortality in the seed pool or diapausing animals during the period of dormancy.

As a transition to examining stochastic variation in the environment, let us examine the situation in which the environment cycles include more than one generation in each environment. Figure 5 gives the region of polymorphism when there two successive, $11221122 \ldots$, and four successive, $11112222 \ldots$, generations in each environment and $g=0.5$. As might be expected, the region of polymorphism is much less with these deterministic environmental patterns. With these cycles, only the individuals that do not go into storage can sometimes avoid the unfavourable environment. For example, with a two-generation cycle and assuming that $A_{2} A_{2}$ has a high fitness in environment 1 , only when environment 1 is followed by environ-

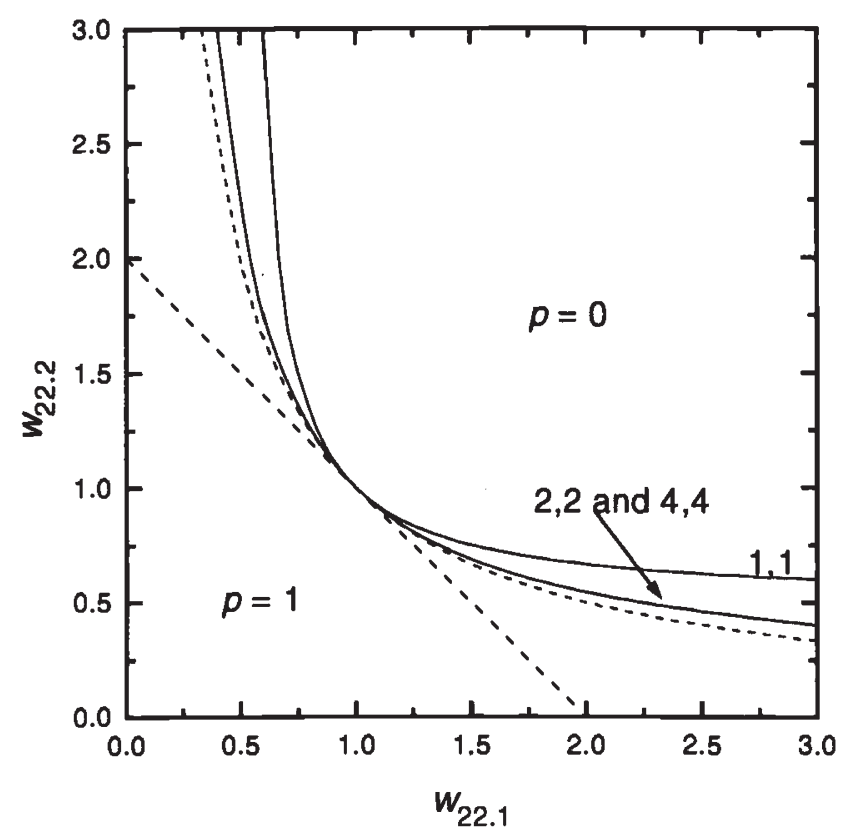

Fig. 5 Region of stability when the fitness of $A_{2} A_{2}$ varies between $w_{22,1}$ and $w_{22.2}$ in a cyclic manner every generation (solid line) or every two or four generations (dotted line) and the level of delayed germination or diapause is $g=0.5$. The conditions when there are either two or four generation cycles are so similar that they are represented by one line. The regions where allele $A_{1}$ is lost or fixed are indicated by $p=0$ and $p=1$, respectively. ment 1 do the alleles in $A_{2} A_{2}$ temporarily escape environment 2 .

\section{Stochastic variation in the environment}

The effect of stochastic variation in the environment on the conditions for stability can best be shown by examining a case with a given $g$ level and specific fitness values which give stability when there is a cyclic environment and alternation of generations in the two environments every generation, that is, $r=-1$. For example, if $g=0.5, w_{22,1}=1.5$ and $w_{22.2}=0.55$, then the mean equilibrium (over both environments) with $r=-1$ is 0.668 . However, if the level of autocorrelation increases, the mean is reduced and the distribution becomes somewhat wider (Fig. 6). The means and variances (in parentheses) for this example when $r=-0.75,-0.5$ and -0.25 are $0.519(0.0053)$, $0.365(0.0103)$ and $0.185(0.0113)$, respectively. Notice the bimodality of the distribution when $r=-0.75$ resulting from the high likelihood of switching between the two environments. If $r=0$, the mean is 0.000 and the polymorphism is lost. In other words, the polymorphism which is present when there is high negative correlation in the environment, is lost when the autocorrelation is increased.

As a second example, the stable equilibrium distribution for the case when $g=0.25, w_{22.1}=1.5$ and $w_{22.2}=0.4$ is given in Fig. 7. When $r=-1$ for these

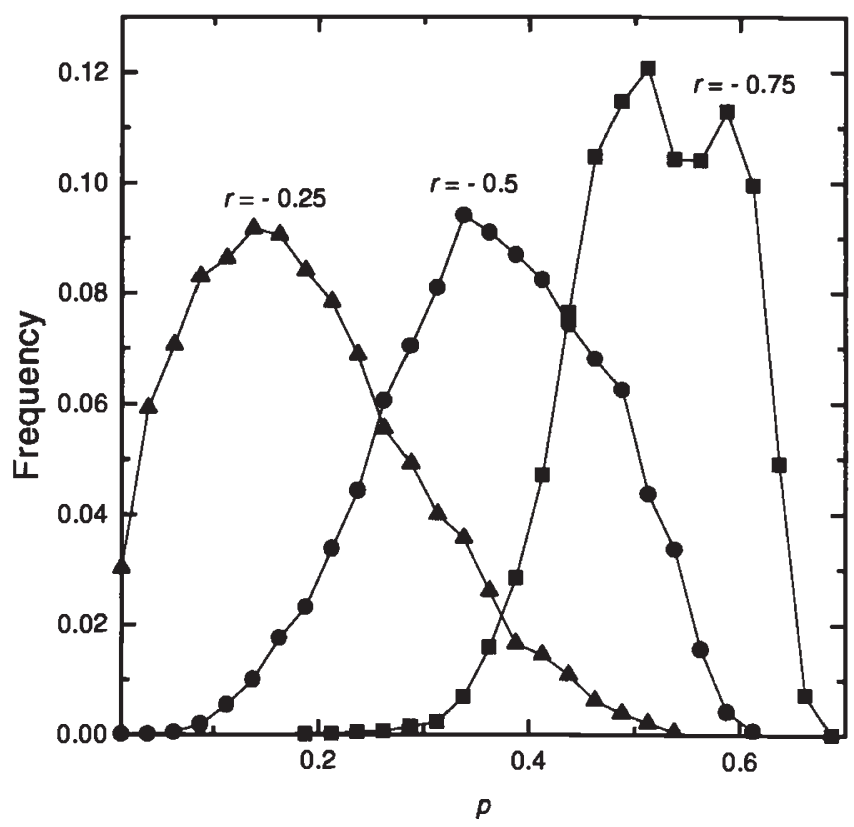

Fig. 6 Equilibrium distribution of the frequency of $A_{1}$ when the autocorrelation between environments is $-0.75,-0.5$ or -0.25 . The mean stable equilibrium over the two environments when $r=-1$ is 0.668 . 


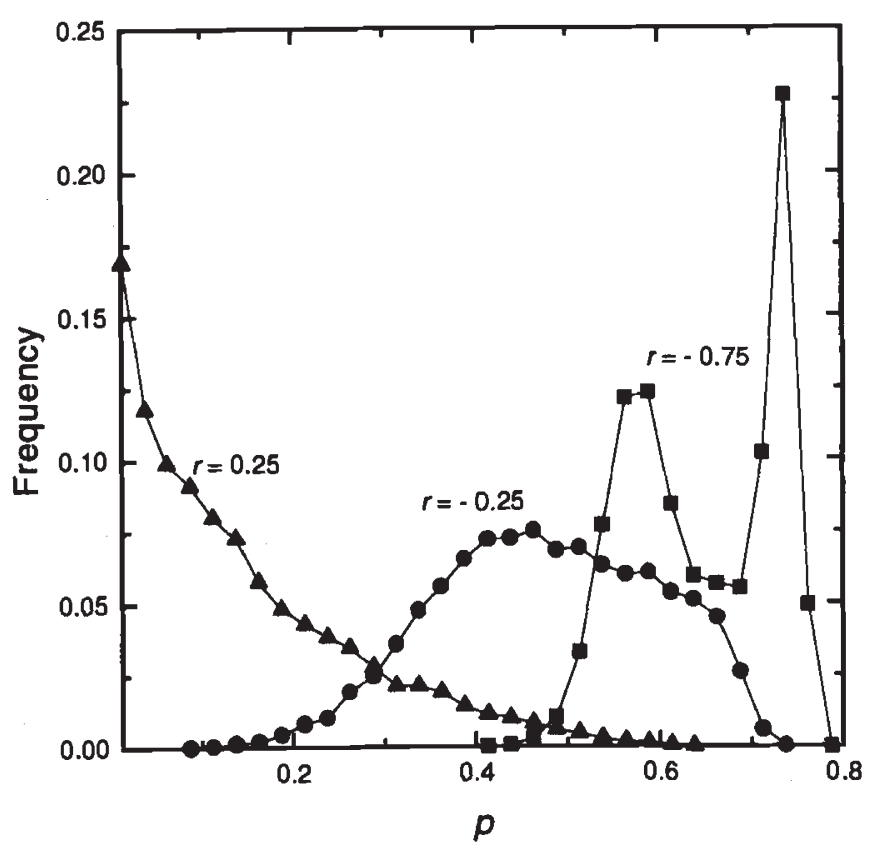

Fig. 7 Equilibrium distribution of the frequency of $A_{1}$ when the autocorrelation between environments is $-0.75,-0.25$ or 0.25 . The mean stable equilibrium over the two environments when $r=-1$ is 0.624 .

values, there is a stable equilibrium at 0.624 and an unstable equilibrium at 0.895 . On the other hand, the means (and variances) of the stable distribution when $r=-0.75,-0.25$ and 0.25 are $0.647(0.0063)$, $0.477(0.0142)$ and $0.140(0.0151)$, respectively (there does not appear to be any unstable equilibrium for these correlations). As noted above, the change in allele frequency is very slow when the frequency of $A_{1}$ is close to unity because there are few $A_{2} A_{2}$ genotypes present. For $r=0.75$, the mean is 0.000 and there does not appear to be any equilibrium. Notice that the range of autocorrelation for which there is polymorphism is much greater for this combination of parameters in which there is higher delayed germination or diapause than the previous example. Again, for the most negative correlation, $r=-0.75$, there is a distinct bimodality of the equilibrium distribution representing the oscillation between the two different environments.

\section{Discussion}

Spatial variation in the environment is often cited as a more likely mode of maintenance of genetic polymorphism than temporal variation in the environment. However, I have shown here that when an unfavourable environment can be avoided by either delayed germination or diapause, then the conditions for a polymorphism may be significantly increased when there is temporal variation in the environment. This conclusion has been shown to be true for the absolute dominance model in which the heterozygote has the same fitness as one of the homozygotes so that no type of heterozygous advantage is possible (the absolute dominance model is the least likely genetic model for maintenance of polymorphism).

The conditions are broadened most when there is a strict switch between two environments every generation and the proportion of seeds that delay germination or animals that diapause is high. When some of the seeds or animals die during the storage time in delayed germination or diapause, the conditions are not as broad. Furthermore, when the environment has a greater autocorrelation (greater than the -1 value from the deterministic switch every generation), then the conditions for a polymorphism are also lessened. However, when there is high delayed germination or diapause, even with a positive autocorrelation, then polymorphism can be maintained under some situations.

When the degree of delayed germination or diapause is high, then there are two surprising findings. First, the lower bound is no longer the arithmetic mean, as for models with no delayed germination or diapause, but encompasses a much larger region. Secondly, there may be an unstable equilibrium, nearer $p=1$ than the stable equilibrium. However, it is unlikely that this unstable equilibrium is of major significance in determining the allele frequency when there is not a strict cycle between environments every generation or if there is a finite population size because the change in allelic frequency above this equilibrium is so slight per generation.

There are only a few theoretical studies in which a specific genetic model with delayed germination of diapause has been investigated. For example, Templeton \& Levin (1979) showed that the presence of seed banks generally slows down the rate of genetic change but they did not address the effects of a seed bank on conditions for genetic polymorphism. In their recent study, Ellner \& Hairston (1994) did show that a general model with overlapping generations can maintain genetic variance, given that the variance of selective fluctuations, the generational overlap, and selection intensity are sufficiently high. However, they also did not consider conditions for polymorphism for specific loci.

In one sense, I have considered what is probably the optimum situation for genetic polymorphism when there is delayed germination or diapause in temporally variable environments because of the general emphasis in the literature on the greater relative importance of 
spatial environmental variation. I have considered only two environments and that the individuals in the seed bank or those diapausing emerge in the subsequent or second generation. As a result, if there is no emergence in the first generation and a strict cyclic environmental change, then the conditions for polymorphism would be greatly broadened as one might intuit from the introductory discussion above concerning escaping unfavourable environments.

It is not clear how often such environmental and germination patterns occur in nature. Venable (1989) gives examples in which seeds from some temperate zone annuals may germinate either in the spring or the fall and that seeds from some desert winter annuals may germinate in October-November or December-January. In both these cases, there is a strict seasonal alteration of the environment and a proportional germination in the next environment and little carryover to beyond 1 year in the seed bank, making these conditions consistent with those that I explored. On the other hand, Hairston \& De Stasio (1988) observed the emergence of a cohort of diapausing copepods over several years. In this latter case and many others concerning the seed banks of plants (Leck et al., 1989), the environmental pattern for the emerging individuals is probably not just two main environmental types as in the examples given by Venable (1989) and the diapause or seed bank emerges over several different environments. Both of these factors, that is, more than two different temporal environments and a longer-lived seed or diapause bank, appear to narrow the conditions for genetic polymorphism (P. W. Hedrick, unpublished data).

There is one type of model that appears to broaden the polymorphism conditions even more than the one I explored. This is a genetic model in which different genotypes have different emergence schedules, and in which they tend to emerge into an environment in which they have an increased fitness (P. W. Hedrick, unpublished data). In this case, as in the situation in which specific genotypes select habitats for which they are adapted (Hedrick, 1990a,b), there are much broader conditions for polymorphism than when there is no genotypic difference for emergence into specific environments.

\section{Acknowledgements}

I appreciate the comments of Nelson Hairston and Michael Turelli on the manuscript and discussions with John Gillespie, Michael Turelli and Larry Venable on the models that I investigated.

\section{References}

CHESSON, P. L. 1983. Coexistence of competitors in a stochastic environment: the storage effect. Lec. Notes in Biomath., 54, 188-198.

CHESSON, P. L. AND WARNER, R. R. 1981. Environmental variability promotes coexistence in lottery competitive system. Am. Nat., 117, 923-943.

CHRISTIANSEN, F. B. 1974. Sufficient conditions for protected polymorphism in a subdivided population. Am. Nat., 108, 157-166.

DEMPSTER, E. 1955. Maintenance of genetic heterogeneity. Cold Spring Harb. Symp. Quant. Biol., 20, 25-32.

ELNER, S. AND HAIRSTON, N. G. JR. 1994. Role of overlapping generations in maintaining genetic variation in a fluctuating environment. Am. Nat., 14, 403-417.

FRANK, S. A. AND SLATKIN, M. 1990. Evolution in a variable environment. Am. Nat., 136, 244-260.

GILLESPIE, J. H. 1976. A general model to account for enzyme variation in natural populations. II. Characterization of the fitness functions. Am. Nat., 110, 809-821.

HAIRSTON, N. G. AND DE STASIO, B. T. 1988. Rate of evolution slowed by a dormant propagule pool. Nature, 336, 239-242.

HEDRICK, P. W. 1986. Genetic polymorphism in heterogeneous environments: a decade later. Ann. Rev. Ecol. Syst., 17, 535-566.

HEDRICK, P. w. 1990a. Theoretical analysis of habitat selection and the maintenance of genetic variation. In: Barker, J. S. F., Starmer, W. T. and MacIntyre, R. J. (eds) Ecological and Evolutionary Genetics of Drosophila, pp. 209-227. Plenum Press, New York.

HEDRICK, P. W. 1990b. Genotypic-specific habitat selection: a new model and its application. Heredity, 65, 145-149.

HEDRICK, P. W., GINEVAN, M. E. AND EWING, E. P. 1976. Genetic polymorphism in heterogeneous environments. Ann. Rev. Ecol. Syst., 7, 1-33.

hOeKstRA, R. F., BIJlsma, R. AND DOlman, A. J. 1985. Polymorphism from environmental heterogeneity: models are only robust if the heterozygote is close in fitness to the favoured homozygote in each environment. Genet. Res., 45, 299-314.

LECK, M. A., PARKER, V. T. AND SIMPSON, R. L. (eds) 1989. Ecology of Seed Banks. Academic Press, New York.

LEVENE, H. 1953. Genetic polymorphism when more than one ecological niche is available. Am. Nat., 87, 331-333.

PROUT, T. 1968. Sufficient conditions for multiple niche polymorphisms. Am. Nat., 102, 493-496.

SEGER, J. AND BROCKMAN, H. J. 1987. What is bet-hedging? Oxford Surv. Evol. Biol., 4, 182-211.

SHMIDA, A. AND ELLNER, S. 1984. Coexistence of plant species with similar niches. Vegetatio, 58, 29-55.

TEMPLETON, A. R. AND LEVIN, D. A. 1979. Evolutionary consequences of seed pools. Am. Nat., 114, 232-249.

VENABLE, D. L. 1989. Modeling the evolutionary ecology of seed banks. In: Leck, M. A., Parker, V. T. and Simpson, R. L. (eds) Ecology of Seed Banks, pp. 67-87. Academic Press, New York. 